\title{
Boots on the Ground, Both Hands on the Keyboard: Harnessing the Power of Resident as Teacher Hybrid Teaching Skills in the Midst of the COVID-19 Pandemic
}

\author{
Scott Moerdler $^{1}$ (D) $\cdot$ Marguerite Costich $^{2} \cdot$ Emily Avis Redwood $^{2} \cdot$ Marina Catallozzi $^{3} \cdot$ Suzanne Friedman $^{2}$
}

Accepted: 3 December 2020 / Published online: 20 January 2021

(C) International Association of Medical Science Educators 2021

Resident as teacher (RAT) programs have grown nationally with much documented success [1], in large part because of their emphasis on "near-peer" teaching and learning [2]. Residents can relate to fellow residents and medical students, as well as be more attentive to challenges and stresses because of shared experiences and proximity in training [3-5]. Residents enjoy teaching and report greater job satisfaction when given teaching opportunities [6, 7]. Efforts focused on training residents to develop and hone their teaching skills have tremendous potential benefits for learners and teachers, and never has this been more apparent than in the current educational climate.

The COVID-19 pandemic has upended graduate medical education (GME). Residents have flexed into different roles to meet institutional demands and traditional modalities for teaching and learning have, in some sense, been abandoned due to social distancing guidelines put in place to limit transmission of disease. The Accreditation Council for Graduate Medical Education has relaxed some requirements, allowing for greater program flexibility, but there is mounting concern that this comes at a cost, threatening the mission of trainee education and ultimately trainee competency [8]. Throughout the pandemic, residents have demonstrated remarkable resilience while providing exceptional patient care. As we move

Scott Moerdler

Scott.moerdler@rutgers.edu

1 Division of Pediatric Hematology Oncology, Rutgers Cancer Institute of New Jersey, Rutgers Robert Wood Johnson Medical School, 195 Little Albany St, New Brunswick, NJ, USA

2 Department of Pediatrics, Columbia University Medical Center, New York, NY, USA

3 Departments of Pediatrics and Population and Family Health, Columbia University Medical Center, NY New York, USA forward into the subsequent pandemic phases, we must prepare for the next challenge of prioritizing and reimagining resident education. We must empower residents to serve as educational leaders in this changed medical landscape by strengthening our RAT programs. These enhanced programs can equip residents with the skills necessary to advance education in this new environment.

In-person teaching has been disrupted. Numerous publications have described the transition from in-person didactics to "remote" learning and need for increased reliance on technology and simulation. Our experiences early in this pandemic have taught us that we cannot simply use virtual platforms to deliver previously in-person lectures and learning experiences. Substituting prior in-person didactics often resulted in a lack of participation and decreased interactions through the screen. In recent months, there has been extraordinary innovation as we have thought of new ways to increase remote learner engagement. These efforts have largely been faculty driven. We now need to engage residents who already have their "boots on the ground" and provide them with additional tools and training to be effective in this new environment. We must improve the long-term sustainability of these innovative models by instilling the fundamental principles of interactive and hybrid learning in residents who are now building their teaching portfolios. To this end, we describe specific techniques related to bedside teaching and incorporation of technology to assist residents in this changed educational landscape. We also describe how employing these techniques may ultimately improve resident wellness and contribute to future sustainability of RAT programs.

\section{Bedside Teaching}

Challenge: Substantial changes to rounding structure and limitations to in-person visit volumes have resulted in fewer 
opportunities to actively engage with residents and students at the bedside.

\section{Proposed solutions:}

- Many programs have had success with residents sharing physical exam findings with fellow residents and medical students using Zoom. For example, "Tele-Rounds" with one learner in the patient room and the remainder of the team outside watching virtually, allowing them to watch and learn the encounter.

- Some faculty have implemented "Learn it and teach it to the next," a concept whereby faculty teach a topic to one small group of residents on rounds, who are then expected to teach that topic to another group of residents not present. By formally giving residents responsibility to teach and share information with their peers, we are empowering them as educators. Faculty can then revisit topics reviewed to assess knowledge acquisition among all residents through questioning, modeling another teaching tool that residents can incorporate into their own teaching practice.

Challenge: The level of acuity of patients seen on the floor may have also changed, necessitating a renewed focus on escalation of care skills.

Proposed solution: Residents can lead simulation exercises and worst-case scenario preparation for other residents, facilitating small group practice while maintaining social distancing. In these teaching scenarios, residents can engage one another through metacognition and further develop clinical reasoning skills necessary for situations requiring a potential escalation of care [9].

\section{Technology and Engagement}

Challenge: The necessity for physical distancing disrupts classic resident teaching forums such as chalk talks and morning reports.

Proposed solutions: Residents are uniquely positioned to think creatively about how to engage across multiple platforms, as they are attuned to the daily resident responsibilities and desire for supplemental education. Advances in technology afford opportunities for even wider resident participation than seen previously.

- Residents have worked with faculty to implement hybrid curricula for residents working remotely, creating additional opportunities to review content and learning/ teaching models with faculty. This practice promotes increased faculty engagement in resident learning, albeit at a physical distance, and can be continued post-pandemic and employed for RAT training.
- Residents have become more comfortable using dynamic, asynchronous modalities, such as online game-based learning platforms (i.e., Kahoot) or social media accounts (e.g., Twitter, Instagram) to engage residents and students learning remotely.

- Greater faculty attendance at virtual conferences or engagement on asynchronous learning platforms allows for greater faculty participation with robust discussions and modeling of best educational practices.

- Residents report back to their peers via email or social media with "pearls" from conferences further to increase engagement among remote learners.

Challenge: Simultaneous engagement of both a small group of in-person learners and a larger group of remote learners poses a challenge which necessitates continued creativity and training [10].

Proposed solutions: We have found that resident-led online games with interactive question-and-answer and case-based teaching with breakout rooms for remote learners on web-based applications work well with both in-person and remote learners. Chat features on streaming platforms, social media use for pictures, videos on teaching points, and virtual games allow for communication and exchange of ideas remotely, while creating a fun and engaging learning experience [11, 12]. Online teaching platforms have created new opportunities for residents to engage and teach one another both in real time and asynchronously.

- $\quad$ These platforms address an urgent and pressing need to maintain a sense of community during these difficult times [13].

- Using these principles, we may be able to create minicommunities of learners, both in-person and virtually, which can help improve social interactions and alleviate feelings of isolation [13].

\section{Conclusion}

The COVID-19 pandemic has challenged us in numerous ways. As resident education has needed to evolve, we have had success in empowering residents to step up and educate each other as well as medical students. With encouragement, modeling, and teaching concrete new RAT skills, we believe residents can elevate their own learning. This has the potential to help develop the next generation of adaptable teachers, build and strengthen resident sense of community, and possibly improve stress and satisfaction during these difficult times. 


\section{References}

1. Hill AG, Yu TC, Barrow M, Hattie J. A systematic review of resident-as-teacher programmes. Med Educ. 2009;43(12):1129-40.

2. Weissman MA, Bensinger L, Koestler JL. Resident as teacher: educating the educators. Mt Sinai J Med. 2006;73(8):1165-9.

3. Knowles MS. Application in continuing education for the health professions: chapter five of "Andragogy in Action". Mobius. 1985;5(2):80-100.

4. Kaufman DM. ABC of learning and teaching in medicine: applying educational theory in practice. BMJ. 2003;326(7382):213-6.

5. Ten Cate O, Durning S. Dimensions and psychology of peer teaching in medical education. Med Teach. 2007;29(6):546-52.

6. Morrison EH, Shapiro JF, Harthill M. Resident doctors' understanding of their roles as clinical teachers. Med Educ. 2005;39(2): 137-44.

7. Ramani S, Mann K, Taylor D, Thampy H. Residents as teachers: near peer learning in clinical work settings: AMEE Guide No. 106. Med Teach. 2016;38(7):642-55.
8. Johnson WR, Blitzer D. Residents' perspectives on graduate medical education during the COVID-19 pandemic and beyond. MedEdPublish. 2020;9.

9. Medina MS, Castleberry AN, Persky AM. Strategies for improving learner metacognition in health professional education. Am J Pharm Educ. 2017;81(4):78.

10. Murdock HM, Penner JC, le S, Nematollahi S. Virtual morning report during COVID-19: a novel model for case-based teaching conferences. Med Educ. 2020;54:851-2.

11. Rose C, Mott S, Alvarez A, Lin M. Physically distant, educationally connected: interactive conferencing in the era of COVID-19. Med Educ. 2020;54:758-9.

12. Moro C, Stromberga Z. Enhancing variety through gamified, interactive learning experiences. Med Educ. 2020;54:1180-1.

13. Rakowsky S, Flashner BM, Doolin J, Reese Z, Shpilsky J, Yang S, et al. Five questions for residency leadership in the time of COVID19: reflections of chief medical residents from an internal medicine program. Acad Med. 2020;95:1152-4.

Publisher's Note Springer Nature remains neutral with regard to jurisdictional claims in published maps and institutional affiliations. 